\title{
Image methodes in chest deformation diagnosis - a case report
}

\author{
Damir Raljević*, Ana Lanča, Irena Kužet-Mioković, Željko Madžar, Davorka Žagar, \\ Vesna Pehar-Pejčinović, Marko Boban, Viktor Peršić \\ Thalassotherapia Opatija - Clinic for treatment, rehabilitation and prevention of cardiovascular \\ disease, Opatija, Croatia
}

\begin{abstract}
Case Report: A 34-year-old patient was admitted to our Institution because of weakness, shortness of breath and exercise intolerance. Echocardiography shows mitral valve prolapse and regurgitation, dilated right ventricle. MSCT coronarography detected changes in the anatomic relations of large blood vessels. The Xiphoideum is drawn back and form pectus excavatum with pressure on the chest structures. No changes in coronary anatomy were found. Cardiac MRI showed that the ventricular systolic function is preserved. No fibrosis was found. This finding reflects the compression-induced changes in anatomy and geometric distortion of the RV. The outflow of large blood vessels is normal, whereas the aorta moved to the left. The exercise test showed a reduced functional capacity. We concluded that there is no structural heart disease and that the symptoms were due to pectus excavatum.
\end{abstract}

Discussion: The patient was admitted to differentiate the etiology between the structural heart disease or the presence of a pectus excavatum. In a large number of cases the symptoms appear in adolescence or early adulthood. Wilting in the thorax can lead to shifts in the thorax structure

Received: $21^{\text {st }}$ Apr 2014

*Address for correspondence: Thalassotherapia Opatija, Maršala Tita 188/1, HR51410 Opatija, Croatia.

Phone: +385-91-5970-296

E-mail: damir.raljevic@gmail.com which may lead to reduced cardiac ejection or disrupt lung function. In the available literature the reduction of functional capacity is well documented. The reduction in functional capacity is caused by a decrease in stroke volume and respiratory capacity. Earlier research has shown the reduction $\mathrm{VO}_{2} \mathrm{max}$ at $75 \%$ predicted for age. Pectus excavatum is associated with mitral valve prolapse and regurgitation with the prevalence of $20 \%$. The changes in cardiac MRI findings are a consequence of compression of the pectus excavatum on structures in the mediastinum with a displacement of the heart to the left with a rotation about the longitudinal axis which leads to the compression of the RV and the change of the anatomical relationship of large blood vessels.

Conclusion: Pectus excavatum, the most common congenital malformations of the chest with the prevalence of 0.1 $-0.3 \%$ of the general population. In a large number of cases the repercussions appear in adolescence or early adulthood which are manifested by reduced exercise tolerance and dyspnea. These problems are caused by cardiac compression and a reduction in stroke volume and restrictive respiratory disorders. Functional testing and imaging methods are necessary together with the clinical picture in assessing the degree of malformation and its repercussions on the cardiovascular system.

KEYWORDS: chest, deformation, heart.

CITATION: Cardiol Croat. 2014;9(5-6):190.

\section{Literature}

1. Rowland T, Moriarty K, Banever G. Effects of pectus excavatum deformity on cardiorespiratory fitness in adolescent boys. Arch Pediatr Adolesc Med. 2005;159:1069-73. 2. Wynn SR, Driscoll DJ, Ostrom NK, et al. Exercise cardiorespiratory function in adolescents with pectus excavatum. Observations before and after operation. J Thorac Cardiovasc Surg. 1990;99:41-7.

3. Schamberger RC, Welch KJ. Incidence of mitral valve prolapse associated with pectus excavatum. J Pediatr. 1987;111:404-7. 